\title{
Study of a quadrupole ion trap with damping force by the two-point one block method.
}

\begin{abstract}
The capabilities and performances of a quadrupole ion trap under damping force based on collisional cooling is of particular importance in high-resolution mass spectrometry and should be analyzed by Mathieu's differential solutions. These solutions describe the stability and instability of the ion's trajectories confined in quadrupole devices. One of the methods for solving Mathieu's differential equation is a two-point one block method. In this case, Mathieu's stability diagram, trapping parameters $\mathrm{a}(\mathrm{z})$ and $\mathrm{q}(\mathrm{z})$ and the secular frequency of the ion motion $\mathrm{w}(\mathrm{z})$, can be derived in a precise manner. The two-point one block method (TPOBM) of Adams Moulton type is presented to study these parameters with and without the effect of damping force and compared to the 5th-order Runge-Kutta method (RKM5). The simulated results show that the TPOBM is more accurate and 10 times faster than the RKM5. The physical properties of the confined ions in the $\mathrm{r}$ and $\mathrm{z}$ axes are illustrated and the fractional mass resolutions $\mathrm{m} / \Delta \mathrm{m}$ of the confined ions in the first stability region were analyzed by the RKM5 and the TPOBM. The Lagrange interpolation polynomial was applied in the derivation of the proposed method. The proposed method will be utilized to obtain a series solution directly without reducing it to first order equations. The problem was tested with the ion trajectories in real time with and without the effect of damping force using constant step size. Numerical results from the two-point one block method have been compared with the fifth order Runge-Kutta method. The proposed two-point one block method has a potential application to solve complicated linear and nonlinear equations of the charged particle confinement in a quadrupole field especially in fine tuning accelerators, and, generally speaking, in physics of high energy.
\end{abstract}

Keyword: Block method; Quadrupol ion trap. 